\title{
RELACIÓN ENTRE EL CRECIMIENTO ECONÓMICO Y LA PRODUCTIVIDAD DEL FACTOR TRABAJO EN GUATEMALA DE 1995 A 2017
}

Reseña de tesis

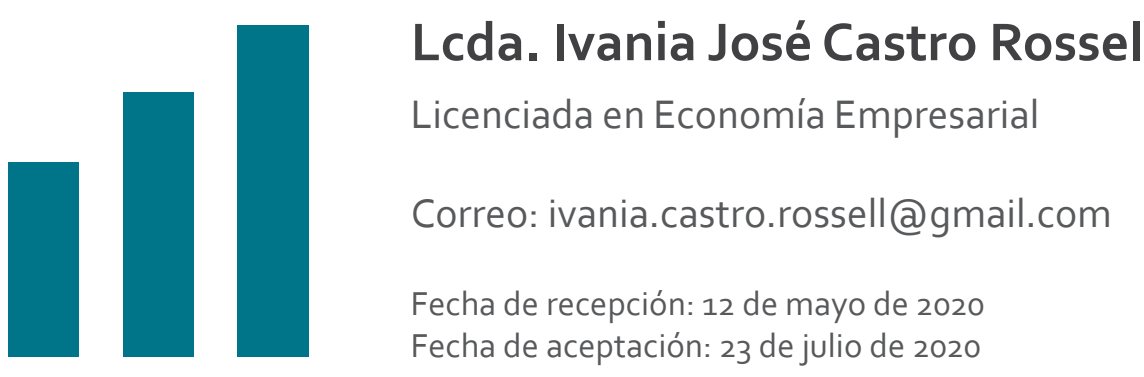

\section{Resumen}

La presente investigación utiliza la metodología expuesta en las leyes de Okun (1962) y KaldorVerdoorn (1966): primero, para identificar el efecto de corto plazo que puede presentar el producto sobre el nivel de desempleo, y segundo, para estimar si efectivamente el producto puede explicar el comportamiento de la productividad laboral, o si, en efecto, otros factores ajenos al indicado pueden tener relación con el comportamiento de dicha productividad.

Es por ello que este estudio propone comprobar ambas causalidades a través de la aplicación de mínimos cuadrados ordinarios, con el propósito de presentar una evidencia empírica y significativa que permita comprobar que el crecimiento económico sí puede ser un determinante de la productividad del factor trabajo en Guatemala, desde 1995 a 2017.

Luego de emplear la metodología descrita, el estudio concluye que variables ajenas al crecimiento económico pueden describir de forma significativa el comportamiento de las tasas de crecimiento de la productividad del factor trabajo en Guatemala, durante el período de análisis establecido.

Palabras clave: productividad laboral, crecimiento económico, desempleo, keynesianismo, mínimos cuadrados ordinarios 


\title{
RELATIONSHIP BETWEEN ECONOMIC GROWTH AND LABOR PRODUCTIVITY IN GUATEMALA FROM 1995 TO 2017
}

Thesis Review

\begin{abstract}
The present investigation uses a methodology based on the Okun (1962) and KaldorVerdoorn (1966) laws - first, in order to identify the short term effects that a product may have on unemployment levels; and second, to estimate if said product can effectively explain how labor productivity behaves; or if indeed, there are other factors that somehow impact productivity behavior.

This study pretends to prove both causalities through the ordinary least squares application, with the purpose of presenting significant and empirical evidence to prove that economic growth could have been a determinant of labor productivity in Guatemala from 1995 to 2017.

After using the previously mentioned methodology, the study concludes that certain variables unrelated to economic growth can describe in important ways the behavior of labor productivity in Guatemala during the referred time period.
\end{abstract}

Keywords: labor productivity, economic growth, unemployment, Keynesian economics, ordinary least squares 


\section{Introducción}

La búsqueda científica y empírica de variables que logren explicar el crecimiento económico de un país, ha sido parte de la historia económica presentada en las diversas teorías desarrolladas desde los clásicos hasta autores más recientes. Dentro de las mismas, figuran las causalidades desarrolladas por escuelas neoclásicas y keynesianas, que tienen como objetivo principal explicar el comportamiento de la productividad laboral a través del crecimiento económico.

Es por ello que el presente trabajo planteó como objetivo principal determinar el nivel de incidencia que puede presentar el comportamiento del crecimiento económico en la productividad del factor trabajo en Guatemala, durante el período correspondiente de 1995 a 2017.

La metodología utilizada describe como sujeto de investigación las observaciones vinculadas con las tasas de desempleo abierto y las tasas de crecimiento de la productividad del factor trabajo, tomando como unidad de análisis las tasas de crecimiento del Producto Interno Bruto (PIB) y las tasas de desempleo que no son explicadas por el crecimiento del PIB.

Estas razones permiten emplear un modelo estimado en dos fases, con base en las leyes de Okun (1962) y Kaldor-Verdoorn (1966), para explicar de forma significativa el comportamiento de la tasa de crecimiento de la productividad del factor trabajo en Guatemala durante el período de análisis. 


\section{Revisión literaria}

Villavicencio y Ochoa (2017) indicaron que una forma en la que se ha analizado la relación inversa entre el crecimiento económico y el desempleo ha sido a través de la ley de Okun (1962), la cual ha sido ampliamente aceptada en la teoría macroeconómica.

Teóricamente, la ley de Okun (1962) establece que una economía en crecimiento, con una población económicamente activa (PEA) estable a lo largo de cierto tiempo, debe aumentar el volumen de recurso humano empleado para incrementar el nivel de producción, acotando al mismo tiempo el nivel de desempleo existente en dicha economía. De igual forma, el mismo autor indica que, en el caso de economías que presenten recesión, pueden observarse mayores tasas de desempleo derivado de significativas disminuciones en la PEA de los países.

La forma básica para medir esta relación, según Okun (1962), es a través del análisis de las diferencias entre el crecimiento en la tasa de desempleo y el crecimiento del $\mathrm{PIB}$, como se describe a continuación:

Donde:

$$
\Delta U n r t=\varphi-\beta_{1} g t(1)
$$

$\Delta U n r t=$ cambios en la tasa de desempleo

$\varphi=$ una constante que indica los cambios exógenos en el crecimiento de la tasa de desempleo

$g t=$ el crecimiento del PIB

El parámetro $\beta$ es conocido como el coeficiente de Okun (1962), el cual integra la correlación del crecimiento del producto y los movimientos de desempleo (Miranda, 2016).

Por otra parte, Kaldor (1966) desarrolló tres leyes de crecimiento económico endógeno. La primera es la alta correlación existente entre el crecimiento del producto industrial y el crecimiento del PIB; la segunda establece la estrecha relación entre la tasa de crecimiento de la productividad industrial y el crecimiento del producto manufacturero; la tercera, es la relación positiva entre el crecimiento de la productividad total de la economía y el crecimiento del sector industrial, y la relación negativa con el incremento del empleo en los sectores no manufactureros.

La relación de la segunda ley también se conoce como ley de Verdoorn (1949), en donde un coeficiente menor a uno indica rendimientos crecientes a escala. Moreno (2008) indica que algunos autores sostienen que esta causalidad va en sentido 
contrario, es decir, del crecimiento de la productividad al crecimiento del producto industrial, y afirman que se debe a la importancia de la brecha tecnológica en la definición de la productividad.

Desarrollando la segunda ley de Kaldor (1966) en Angeriz, McCombi \& Roberts (2008), la ley de Verdoorn (1949) original se puede estimar como:

$$
p_{j}=c+\beta q_{j}(2)
$$

Donde:

$p_{j}=$ la tasa de crecimiento de la productividad del trabajo

$q_{j}=$ el producto manufacturero respectivamente para una región $j$

El coeficiente de Verdoorn (1949) es el parámetro $\beta$, el cual se espera que sea positivo e inferior a la unidad. De esta forma, se espera que exista una relación positiva entre el incremento del empleo y el producto dentro del sector industrial (Sánchez y García, 2015).

\section{Aplicación de las leyes de Okun y Kaldor-Verdoorn para el caso guatemalteco}

Para identificar si, en efecto, el crecimiento económico puede explicar de manera significativa el comportamiento de la tasa de desempleo abierta y la productividad laboral desde 1995 a 2017 en Guatemala, primero, se estimaron ambas leyes de manera individual para luego proceder a su aplicación conjunta. A continuación, se presentan los instrumentos utilizados para la evaluación de las leyes de forma individual:

$$
\begin{gathered}
U_{n t}=\Omega+\beta_{i} g_{t}+\beta_{i} d_{t}+u_{t}(\text { (3) } \\
P g_{t}=\propto+\beta_{i} g_{t}+\varepsilon_{t}(4)
\end{gathered}
$$

Donde:

$U_{n t}=$ crecimiento de la tasa de desempleo

$\Omega=$ constante de estimación

$g_{t}=$ crecimiento del producto

$d_{t}=$ variable dicotómica

$u_{t}=$ término de perturbación aleatoria que se distribuye normalmente $\left(0, \sigma^{2}\right)$

$P g_{t}=$ crecimiento de la tasa de productividad del factor trabajo

$\propto=$ constante de estimación

$\varepsilon t=$ término de perturbación aleatoria que se distribuye normalmente $\left(0, \sigma^{2}\right)$ 
En el caso de la aplicación de las leyes de Okun (1962) y Kaldor-Verdoorn (1966) de forma conjunta, la adaptación del modelo propuesto se compuso por dos fases. En la primera fase, se utilizaron variables que no estuviesen correlacionadas con el término de error, para calcular los valores estimados de dicho término. En la segunda fase, se utilizaron los valores estimados del término de error de la primera fase para estimar un modelo de regresión lineal para la variable dependiente, crecimiento de la productividad del factor trabajo, tal como se presenta a continuación:

$$
\begin{gathered}
U_{n t}=\Omega+\beta_{i} g_{t}+u_{t}(5) \\
P g_{t}=\propto+\beta_{1} g_{t}+\beta_{2} \hat{u}_{t}+\beta_{3} d_{t}+\varepsilon_{t}(6)
\end{gathered}
$$

Donde:

$U_{n t}=$ crecimiento de la tasa de desempleo

$\Omega=$ constante de estimación

$g_{t}=$ crecimiento del producto

$u_{t}=$ término de perturbación aleatoria que se distribuye normalmente $(0$,

$P g_{t}=$ crecimiento de la tasa de productividad del factor trabajo

$\propto=$ constante de estimación

$u t=$ componente de residuo (estimado en la ecuación 5)

$d_{t}=$ variable dicotómica

$\varepsilon_{t}=$ término de perturbación aleatoria que se distribuye normalmente $(0$,

De esta forma, tal como establecen Amico, Fiorito y Hang (2011), la ecuación 6 tendría valores no sesgados por la omisión de variables significativas para los $\beta_{1}$ y $\beta_{2}$ del modelo, ya que el $\beta_{1}$ reflejaría los efectos estructurales que el crecimiento económico tiene sobre la productividad laboral. Por otro lado, el impacto que tiene el desempleo que no se ve afectado por el crecimiento del producto, estaría presente en los errores estimados de la ecuación 6 , crecimiento de la tasa de productividad del factor trabajo, representados como $\beta_{2}$.

Los resultados obtenidos en la aplicación individual de la ley de Okun (1962) reflejaron que, con el supuesto de que el término de perturbación se distribuye normalmente, existe una relación negativa entre el crecimiento de la tasa de desempleo y el crecimiento económico del PIB, ya que se obtuvo un parámetro de -22.30, expresando que por cada punto porcentual que incremente el crecimiento económico, en promedio, existe una disminución de la tasa de desempleo de $22.30 \%$.

Utilizando la prueba de p-valor a un nivel de significancia del $5 \%$, se pudo confirmar que la tasa de crecimiento del PIB no explica de forma significativa las variaciones 
de la tasa de desempleo del país durante el período sujeto a análisis. Por otro lado, utilizando la probabilidad del estadístico $F$, también con un nivel de significancia del $5 \%$, se determinó que, en conjunto, las variables independientes no explican significativamente el modelo econométrico.

\section{Tabla 1}

Resumen de resultados de estimación de ley de Okun para Guatemala

\begin{tabular}{|l|l|l|l|}
\hline Variable dependiente & \multicolumn{2}{|c|}{ Tasa de crecimiento del desempleo } \\
\hline Coeficiente de crecimiento económico & -22.30 & Probabilidad & 0.29 \\
\hline Coeficiente dicotómico (2009) & 79.60 & Probabilidad & 0.88 \\
\hline Coeficiente de determinación múltiple & 0.06 & $\begin{array}{l}\text { Probabilidad } \\
\text { estadístico F }\end{array}$ & 0.54 \\
\hline Estadístico de Durbin Watson & & 1.20 & \\
\hline
\end{tabular}

Fuente: elaboración propia a través del programa estadístico Eviews 8

Este resultado se obtuvo aun con la inclusión de una variable dicotómica que absorbía el cambio estructural presentado por la economía guatemalteca en el 2009, producto de la última crisis económica de Estados Unidos, que afectó significativamente tanto la tasa de desempleo como el crecimiento del PIB guatemalteco.

En el caso de la aplicación individual de la ley de Kaldor-Verdoorn (1966), respetando el mismo supuesto de normalidad de los errores, se obtuvo un valor de 0.98 , lo cual estableció que por cada punto porcentual que incrementara el crecimiento económico, en promedio, la productividad del factor trabajo incrementaba $0.98 \%$.

Empleando la prueba de p-valor a un nivel de significancia del $5 \%$, se pudo aceptar que la tasa de crecimiento del PIB explicaba de forma significativa las variaciones de la tasa de productividad laboral del país durante el período sujeto a análisis. De igual forma, el valor de la probabilidad del estadístico $F$, a un nivel de significancia del $5 \%$, estableció que la variable independiente explicaba significativamente el modelo econométrico. 


\section{Tabla 2}

Resumen de resultados de estimación de la ley de Kaldor-Verdoorn para Guatemala

\begin{tabular}{|l|l|l|l|}
\hline Variable dependiente & \multicolumn{2}{|c|}{ Productividad del factor trabajo } \\
\hline Coeficiente de crecimiento económico & 0.98 & Probabilidad & 0.00 \\
\hline Coeficiente de determinación múltiple & 0.99 & $\begin{array}{l}\text { Probabilidad } \\
\text { estadístico F }\end{array}$ & 0.00 \\
\hline Estadístico de Durbin Watson & \multicolumn{2}{|c|}{0.17} \\
\hline
\end{tabular}

Fuente: elaboración propia a través del programa estadístico Eviews 8

Ahora bien, al obtener una perspectiva individual en la aplicación de ambas leyes para el caso guatemalteco, se procedió a sus estimaciones conjuntas, tal como se indicó con anterioridad, para determinar si únicamente el crecimiento económico puede explicar estadísticamente el comportamiento de la tasa de desempleo abierto y la productividad laboral, o si bien, variables ajenas al crecimiento del PIB explican de manera significativa el comportamiento de las variables sujetas a análisis.

En la primera fase de estimación, se evaluó el efecto que tiene la tasa del crecimiento económico sobre la tasa de desempleo en Guatemala. El objetivo de esta primera fase fue la obtención del término de perturbación estocástica, el cual permitía explicar la variación del desempleo que no era explicada por la variación del crecimiento económico.

Los resultados obtenidos en la primera fase establecieron un parámetro para la beta del crecimiento económico de -21.47, con un p-valor de 0.27 . El signo fue el esperado, ya que el incremento de un punto porcentual en el crecimiento económico, en promedio, permitía una disminución en la tasa de desempleo de $21.47 \%$. Este resultado se derivó del aumento experimentado en la demanda efectiva, lo que incrementa la demanda laboral para suplirla. Sin embargo, con un nivel de significancia del $5 \%$, se confirmó que la tasa de crecimiento del PIB no explicaba de forma significativa las variaciones de la tasa de desempleo del país durante los años sujetos a análisis. 


\section{Tabla 3}

Resumen de resultados de primera fase de la estimación simultánea de ley de Okun y Kaldor-Verdoorn para Guatemala

\begin{tabular}{|l|l|l|l|}
\hline Variable dependiente & \multicolumn{2}{|c|}{ Tasa de crecimiento del desempleo } \\
\hline Coeficiente de crecimiento económico & -21.47 & Probabilidad & 0.27 \\
\hline Coeficiente de determinación múltiple & 0.0599 & $\begin{array}{l}\text { Probabilidad } \\
\text { estadístico F }\end{array}$ & 0.27 \\
\hline Estadístico de Durbin Watson & \multicolumn{2}{|c|}{1.19} \\
\hline
\end{tabular}

Fuente: elaboración propia a través del programa estadístico Eviews 8

Por otro lado, tal como se estimó en las leyes de Okun (1962) y Kaldor-Verdoorn (1966) por separado, el valor de la probabilidad del estadístico F, a un nivel de significancia del $5 \%$, estableció que la variable independiente no explicaba de forma significativa el crecimiento del desempleo en la primera fase de estimación, lo que sugiere la añadidura de más variables independientes que expliquen de forma significativa el comportamiento de la tasa de desempleo observada durante los años analizados.

El coeficiente de determinación múltiple también confirmó la estimación resultante, ya que la tasa de crecimiento económico explicaba únicamente en un 5.99\% las variaciones resultantes en la tasa de crecimiento del desempleo correspondiente de 1995 a 2017. De igual forma, al comparar las estimaciones realizadas en la ley de Okun (1962), estimada en la Tabla 1 con los resultados de la primera fase, se pudo observar que el modelo resultante en la primera etapa, sin la inclusión de una variable dicotómica por el quiebre estructural producto de la última crisis económica, es el modelo que proporciona la aproximación más cercana a la realidad, según los criterios de información de Akaike y bayesiano.

En la segunda fase, se utilizó la tasa de crecimiento económico y los residuos obtenidos en la primera fase del modelo conjunto como variables explicativas de la productividad del factor trabajo. Dichos residuos buscaron explicar los efectos que diversas variables, ajenas a la tasa de crecimiento económico, podían tener sobre el desempleo del país.

Adicional a ello, en esta fase se agregó una variable dummy, la cual absorbió el efecto de la última crisis económica mundial, corrigiendo el problema de quiebre estructural en la relación de las variables indicadas para el 2009.

Los resultados obtenidos en la segunda fase establecieron un parámetro para la beta del crecimiento económico de -0.02, con un p-valor de 0.99, lo cual indicó que al incrementar el crecimiento económico un punto porcentual, en promedio, 
la productividad del factor trabajo presentaba una disminución del $2 \%$. Es por ello que con un nivel de significancia del $5 \%$, se aceptó la hipótesis nula de que el crecimiento económico no mostraba una relación significativa para explicar el comportamiento de la productividad del factor trabajo en Guatemala desde 1995 a 2017.

\section{Tabla 4}

Resumen de resultados de segunda fase de la estimación simultánea de la ley de Okun y Kaldor-Verdoorn para Guatemala

\begin{tabular}{|l|l|l|l|}
\hline Variable dependiente & \multicolumn{2}{|l|}{ Productividad del factor trabajo } \\
\hline Coeficiente de crecimiento económico & -0.02 & Probabilidad & 0.99 \\
\hline Componente residuo (primera fase) & 0.08 & Probabilidad & 0.00 \\
\hline Coeficiente dicotómica (2009) & -23.47 & Probabilidad & 0.60 \\
\hline Coeficiente de determinación múltiple & 0.51 & $\begin{array}{l}\text { Probabilidad } \\
\text { estadístico F }\end{array}$ & 0.00 \\
\hline Estadístico de Durbin Watson & & \multicolumn{2}{|c|}{2.13} \\
\hline
\end{tabular}

Fuente: elaboración propia a través del programa estadístico Eviews 8

Sin embargo, con un nivel de significancia del $5 \%$, se confirmó que los residuos estimados en la primera fase explicaban de forma significativa el efecto que las variaciones del desempleo presentaban sobre la productividad del factor trabajo en Guatemala desde 1995 a 2017, ya que esta última incrementaba en la medida en que las condiciones de desempleo que no eran explicadas por el crecimiento económico se tornaban más adversas.

Los resultados obtenidos en la segunda fase de estimación también se fundamentaron con el coeficiente de determinación múltiple, el cual estableció que las variaciones en el crecimiento económico y en el componente de residuo estimado en la primera fase explicaban el $51 \%$ de las variaciones establecidas en la productividad del factor trabajo en Guatemala.

Dado que el crecimiento económico no puede explicar el comportamiento de las variables sujetas a análisis, como ejercicio complementario se buscó explicar el comportamiento de la tasa de desempleo abierta y la productividad laboral a través del análisis de variables independientes, como remesas familiares y nivel educativo de la población guatemalteca, durante el mismo período sujeto a análisis, obteniendo los siguientes resultados. 


\section{Tabla 5}

Resumen de estimación del desempleo en función de remesas familiares (1995-2017)

\begin{tabular}{|l|l|l|l|}
\hline Variable dependiente & \multicolumn{2}{|c|}{ Tasa de desempleo abierto } \\
\hline Coeficiente de remesas familiares & -0.2292 & Probabilidad & 0.9035 \\
\hline Coeficiente de constante de estimación & 31.4812 & Probabilidad & 0.663 \\
\hline
\end{tabular}

Fuente: elaboración propia a través del programa estadístico Eviews 8

En el caso de las remesas familiares, pese a que su crecimiento anual no explica significativamente el comportamiento de la tasa de desempleo abierta desde 1995 a 2017, se puede inferir que a medida que incrementa en un punto porcentual el flujo de remesas familiares en el país, disminuye la tasa de desempleo abierta. En suma, el signo negativo del coeficiente estimado no significativo permite inferir que un flujo constante de remesas familiares puede ser una condición necesaria, pero no suficiente, para disminuir los niveles de desempleo, además de que dichas remesas permiten incrementar la calidad de vida de los beneficiarios, como complemento a los ingresos propios y considerados como resultado de la ocupación primaria en el país.

Por otro lado, el posible impacto de la escolaridad en el comportamiento de la tasa de desempleo y la productividad laboral en Guatemala debe abarcarse desde una perspectiva histórica, tal y como lo establecieron Córica y Ortero (2014), al indicar que las políticas educativas implementadas a partir de 1950 sobre la mayoría de países de América Latina, han permitido que la región transite en un proceso de expansión paulatina en el sistema educativo.

Es por ello que, a partir del 2000, Guatemala estipuló nuevas leyes y normativas jurídicas educativas en donde se considera la expansión de la obligatoriedad de la enseñanza hasta concluir la educación secundaria. De hecho, tal y como lo establece la Ley de Educación Nacional (Congreso de la República de Guatemala, 1991), el sistema educativo obligatorio abarca hasta el nivel medio. Este incremento ha beneficiado en gran medida a los sectores de menores ingresos, aunque su efecto no ha sido suficiente para reducir la disparidad del logro educativo.

Con base en los anuarios educacionales presentados por el Ministerio de Educación de Guatemala (2016), se buscó identificar la posible relación entre la tasa de desempleo y el porcentaje de alumnos aprobados por nivel educativo desde 1995 a 2015, obteniendo los siguientes resultados. 


\section{Tabla 6}

Resumen de resultados de primera fase de la estimación simultánea de ley de Okun y Kaldor-Verdoorn para Guatemala en función del nivel educativo (1995-2015)

\begin{tabular}{|l|l|l|l|}
\hline Variable dependiente & \multicolumn{3}{|c|}{ Tasa de desempleo abierto } \\
\hline Coeficiente de nivel educativo primario & 5479.93 & Probabilidad & 0.01 \\
\hline Coeficiente de nivel educativo básico & -1627.76 & Probabilidad & 0.15 \\
\hline Coeficiente de nivel educativo diversificado & 1612.91 & Probabilidad & 0.17 \\
\hline Coeficiente de constante de estimación & -101.11 & Probabilidad & 0.32 \\
\hline
\end{tabular}

Fuente: elaboración propia a través del programa estadístico Eviews 8

El resultado pudo determinar con un nivel de significancia de $5 \%$ que la única variable que puede explicar a proxy el comportamiento de la tasa de desempleo abierta del país, es el coeficiente del nivel educativo primario, estableciendo que por cada punto porcentual que incrementaba la tasa de estudiantes que culminaban el nivel primario, en promedio, la tasa de desempleo abierta del país incrementaba a 5479.93 personas. Este resultado presentó validez, ya que, como señalan Córica y Ortero (2014), en los países en donde el nivel primario de educación aumenta sin que con ello aumenten niveles superiores, se genera más desempleo al incrementarse la oferta de posibles empleados que apliquen a un trabajo de ingresos bajos o en donde las habilidades a emplear sean únicamente técnicas, generando una sobreoferta en el mercado. De cualquier manera, se exhorta al análisis de las variables de escolaridad con más detenimiento en otros estudios que tengan dicha asociación como principal enfoque.

Con respecto a la productividad laboral, se buscó establecer el posible impacto que la educación presentaba sobre la variable sujeta a análisis, y a través del período de 1995 a 2005, se obtuvieron los siguientes resultados.

\section{Tabla 7}

Resumen de resultados de segunda fase de la estimación simultánea de la ley de Okun y Kaldor-Verdoorn para Guatemala en función del nivel educativo (1995-2015)

\begin{tabular}{|l|l|l|l|}
\hline Variable dependiente & \multicolumn{3}{|c|}{ Productividad del factor trabajo } \\
\hline Coeficiente de nivel educativo primario & -90.25 & Probabilidad & 0.86 \\
\hline Coeficiente de nivel educativo básico & 141.57 & Probabilidad & 0.63 \\
\hline Coeficiente de nivel educativo diversificado & 10.40 & Probabilidad & 0.97 \\
\hline Coeficiente del residuo estimado & 0.029 & Probabilidad & 0.66 \\
\hline Coeficiente de constante de estimación & 4.3249 & Probabilidad & 0.87 \\
\hline
\end{tabular}

Fuente: elaboración propia a través del programa estadístico Eviews 8 
Derivado de los resultados obtenidos, se estableció que la productividad laboral no era explicada de forma significativa por el porcentaje de alumnos aprobados en los diversos niveles educativos. Este resultado confirmó las desfavorables condiciones en materia de inserción laboral que impactan en empleos donde existe un menor nivel de productividad, y en donde, dada la estructura de los puestos disponibles en la demanda laboral, la productividad pueda deberse a otros factores como la experiencia y la acumulación de experiencia (ver Díaz [2003]). 


\section{Conclusiones}

Con base en el objetivo general planteado en la presente investigación, se pudo establecer que el crecimiento económico no es un factor determinante para explicar de forma significativa el comportamiento de la productividad del factor trabajo en Guatemala, en el período comprendido desde 1995 a 2017.

De acuerdo a las estimaciones realizadas en la primera fase del modelo propuesto con base en la ley de Okun (1962) para Guatemala, durante los años sujetos a análisis, no existió evidencia estadística que permitiera rechazar la hipótesis nula de que existía una relación significativa entre el crecimiento económico y la tasa de desempleo abierta del país.

Por tanto, aun cuando se evidencie una mejora en los niveles de crecimiento de la economía, esto no necesariamente se traducirá en una reducción del desempleo, asociando este con otras variables no explicadas. Este resultado se fundamentó con la aplicación individual de la ley de Okun (1962), la cual también estimó el cambio estructural presentado por la última crisis económica que afectó considerablemente a la economía guatemalteca.

La estimación de la segunda fase del modelo con base en la ley Kaldor-Verdoorn (1966) para Guatemala, durante el período analizado, no presentó una relación significativa al buscar asociar una mejora de la productividad del factor trabajo con una expansión del Producto Interno Bruto. En suma, la reducción del desempleo y el aumento de la productividad en Guatemala no están relacionadas con el crecimiento de la economía, considerando además que se estimó de forma individual la ley de Kaldor-Verdoorn (1966), obteniendo también resultados no significativos para el período de análisis establecido.

Sin embargo, las variables ajenas al crecimiento económico que pueden explicar la variación del desempleo, estimadas en la primera fase del modelo y aplicadas como variables explicativas de la productividad del factor trabajo en la segunda fase, sí fueron estadísticamente significativas en la aplicación conjunta de las leyes de Okun (1962) y Kaldor-Verdoorn (1966) para Guatemala, durante los años sujetos a análisis, lo cual conlleva a delimitar la teoría económica que puede llegar a explicar las variaciones de la tasa de desempleo y el impacto de la misma en la productividad laboral del país.

Por lo tanto, con base en los resultados expuestos en el presente estudio, se sugiere la profundización en la investigación del tema y el uso de modelos alternativos para explicar la productividad del factor trabajo en Guatemala, mediante la aplicación de otros modelos empíricos que examinen los posibles factores de demanda que puedan establecer de forma significativa las variaciones de la productividad laboral a lo largo del tiempo. 


\section{Referencias}

Amico, F., Fiorito, A. y Hang, G. (2011). Producto potencial y demanda en el largo plazo: hechos estilizados y reflexiones sobre el caso argentino reciente. Centro de Economía y Finanzas para el desarrollo de la Argentina. http://www.iade.org.ar/system/files/dt35.pdf

Angeriz, A., McCombie, J. \& Roberts, M. (2008). Some New Estimates of Returns to Scale for EU Regional Manufacturing, 1986-2002. Estados Unidos. https://www.researchgate.net/publication/249682507_New_Estimates_ ofReturns_to_Scale_and_Spatial_Spillovers_for_EU_Regional_ Manufacturing_1986-2002/link/56631dado8ae15e7463135b6/download

Congreso de la República de Guatemala. (11 de enero de 1991). Decreto Número 12-91. https://web.oas.org/childhood/ES/Lists/Recursos\%20\%20Planes\%20 Nacionales/Attachments/443/16.\%2oLey\%20de\%2oEducaci\%C3\%B3n.pdf

Córica, A. y Ortero, A. (2014). Educación y Empleo en América Latina. Entre tendencias y alcances. Papeles de Población, (82), 167-201.

Díaz, A. (2003). Los Determinantes del Crecimiento Económico: Comercio Internacional, Convergencia y las Instituciones. México: Plaza y Valdés, S. A. de C. V.

Kaldor, N. (1966). Causes of the slow rate of economic growth of the United Kingdom. Cambridge: Cambridge University.

Ministerio de Educación de Guatemala. (2016). Anuario Estadístico de la educación en Guatemala, Años: 1992 al 2019. http://estadistica.mineduc.gob.gt/Anuario/ home.html\#

Miranda, M. (2016). ¿Puede el crecimiento afectar la productividad? Revista de Economía y Finanzas BCN, 3, 59-90.

Moreno, Á. (2008). Las leyes del desarrollo económico endógeno de Kaldor: el caso colombiano. Economía Institucional, 10(18), 129-147.

Okun, A. (1962). Potential Output: Its measurement and significance. American Statistical Association. Proceedings of the Business and Economic Section, 89-104.

Sánchez, I. y García, R. (2015). Estimación de rendimientos crecientes en las manufacturas regionales mexicanas utilizando la ley de Verdoorn. Revista Iberoamericana de Ciencias, 2(1), 36-50. 
Verdoorn, P. (1949). Factors that determine the growth of labour productivity. New York: MacMillan.

Villavicencio, K. y Ochoa, W. (2017). Ley de Okun. Análisis de la relación entre crecimiento y desempleo para 12 países de América Latina. Revista Publicando, $4(3), 1-15$. 